JGG 2020;68:1-6

doi: 10.36150/2499-6564-296

\section{Oral condition assessment among a nursing home population. Analysis of the association between tooth loss and cognitive impairment: an observational study}

\author{
Giulia Redaelli ${ }^{1}$, Fabrizio Giunco², Pietro Davide Trimarchi ${ }^{3}$, \\ Fabrizio Carini ${ }^{4}$ \\ ${ }^{1}$ Università di Milano-Bicocca, Milan, Italy; ${ }^{2}$ Dipartimento Cronicità, IRCCS Fondazione Don \\ Carlo Gnocchi, Milan, Italy; ${ }^{3}$ IRCCS Fondazione Don Carlo Gnocchi, Milan, Italy; ${ }^{4}$ Dipartimento \\ di Medicina e Chirurgia, Università di Milano-Bicocca, Milan, Italy, Centro Odontoiatrico, IRCCS \\ Fondazione Don Carlo Gnocchi, Milan, Italy
}

Background \& aims. Several studies report that a low number of teeth is strictly associated with a higher risk of cognitive impairment. Aim of this study is to evaluate the oral condition of a nursing home population, in order to clarify the possible association between tooth loss and cognitive decline.

Methods. 444 patients were selected; clinical information were excerpted from their hospital records. Subjects were visited in their nursing home unit, paying particular attention to number of teeth, prosthesis and soft tissues.

Received: February 6, 2019

Accepted: October 11, 2019

\section{Correspondence}

Giulia Redaelli

Università di Milano-Bicocca, piazza dell'Ateneo

Nuovo 1, 20126, Milan, Italy

E-mail: giulia.reds.39@gmail.com

\section{Conflict of interest}

The Authors declare no conflict of interest

How to cite this article: Redaelli $G$, Giunco F, Trimarchi PD, et al. Oral condition assessment among a nursing home population. Analysis of the association between tooth loss and cognitive impairment: an observational study. Journal of Gerontology and Geriatrics 2020;68:1-6. https://doi.org/10.36150/2499-6564-296

C Copyright by Società Italiana di Gerontologia e Geriatria (SIGG)

Results. 378 subjects were visited, 277 with formal diagnosis of dementia and 101 with mild or no impairment in cognition. Age was similar in both group, while education level, MMSE and number of teeth were significantly lower among people with dementia. There was an inverse correlation between age and number of remaining teeth, while MMSE did not seem to be associated with tooth loss. School level was positively associated with number of teeth.

Conclusions. There is a significant difference in number of teeth, school level and MMSE between patients with and without dementia. Maybe due to the high prevalence of people with severe cognitive impairment, a significant association between MMSE and number of teeth was not found. The presence of compromise oral conditions is relevant.

Key words: Alzheimer's disease, epidemiology, oral health, prosthesis, cerebrovascular dementia, teeth, geriatrics, gerontology, nursing home

\section{INTRODUCTION}

The constant increase in the average age is determining an increasing epidemiological relevance of different types of cognitive impairment, in particular dementia. In 2018, about 50 million people suffered from some kind of dementia, with an annual incidence of 9.9 million $^{1}$; over the next 20 years, these numbers will nearly double, up to 152 million in 2050. From a socio-economic point of view, the impact of these conditions is considerable: not only patients and their families, but also all public health
This is an open access article distributed in accordance with the CC-BY-NC-ND (Creative Commons Attribution-NonCommercial-NoDerivatives 4.0 International) license. The article can be used by giving appropriate credit and mentioning the license, but only for non-commercial purposes and only in the original version. For further information: https://creativecommons.org/licenses/by-nc-nd/4.0/deed.en 
is affected ${ }^{2}$. Despite the clinical manifestations have been recognized, risk factors of dementia remain unclear, especially of Alzheimer's disease (involving about two-thirds of the sufferers).

Recently, a growing number of research studies have focused on the link between oral health and cognitive status ${ }^{3}$. In particular, it seems possible to assess the association between tooth loss and an increased risk of developing dementia in the elderly ${ }^{4,5}$.

Several research studies report how the reduced and the difficult chewing activity (following a widespread edentulism) and the state of chronic inflammation (typical of periodontal disease) predispose the patient to an increased risk of developing dementia and losing cerebral volume in the areas devoted to memorisation (hippocampus, caudate nucleus and temporal lobe) ${ }^{6}$. A poor oral health and the resulting dietary poverty are also associated with the weakening of systemic health, which can contribute to the onset and progression of dementia $^{7}$. In preparation for a larger population study, the purpose of the current pilot phase is the evaluation of oral conditions - with particular reference to the number of teeth - in an institutionalized population, comparing the results with the anamnesis taken from clinical documentation. Through the investigation of a large number of patients, the aim of this study is clarifying the correlation between a reduced number of teeth and the level of cognitive decline, considering the possible influence of factors such as age and level of education.

\section{PATIENTS AND METHODS}

\section{Participants}

This study was conducted between November 2017 and April 2018 at the Palazzolo Geriatric Institute (Don Carlo Gnocchi Foundation) in Milan. 444 patients were enrolled in the study, all of them selected from 9 Nursing Home $(\mathrm{NH})$ units and the Alzheimer Special Care Unit of the Institute. During the visits, 66 patients were excluded: 21 for their unstable medical condition and 45 who refused to submit to the study (Fig. 1). From the medical records of every patient, the diagnostic profile and the coexistence of potentially confounding variables were evaluated: upcoming and remote conditions (hypertension, diabetes mellitus compensated or not, stroke), drug therapy, habits (smoking and alcohol abuse), level of education, SOSIA score (indicative for daily living abilities, cognition/behaviour, comorbidities) ${ }^{8}$ and the results obtained with test for cognitive assessment and autonomy (MMSE index and Barthel Index, considering the latest performance). If present, the reports of dental services at the Institute's Dental Centre was considered.

\section{Procedures}

Age and sex were used as demographic characteristics; we defined a low education level as less than 10 years of schooling ${ }^{6}$. We classified the group under evaluation in patients with or without cognitive impairment; among patients with dementia, we made a further distinction based on neurodegenerative disease: Alzheimer's disease, cerebrovascular dementia, dementia of other kind. As regards their achievements in the MMSE, we used the NICE classification (2015) $)^{9}: \geq 27$ points, normal; 26-21 points, mild impairment; $20-10$ points, impairment; $\leq 9$ points, severe impairment. We considered edentulous subjects with no teeth, only root or non-osteointegrated implant prosthesis ${ }^{6}$. The visits took place in the ward, with patient in bed or on a wheelchair. For the inspection of the oral cavity a sterile set (mirror, probe and tweezers) and a tongue depressor were used; each control lasted few minutes. In case of good compliance, a photographic documentation of the oral cavity was taken. For each patients, residual teeth (number of teeth, mobility, integrity, any prosthetic rehabilitations fixed, implants), removable dentures (full or partial, fair or unstable, cleaning) and soft tissues (presence of plaque and Tartar, gum bleeding or typical oral lesions such as candida and hypertrophy of lingual papillae) were evaluated. A written report of the visit was attached to the medical records of patients.

\section{AnALYsis}

Descriptive statistics (mean, median, IQR, kurt) were used to characterize the patients. Analysis of distribution of some data (age, school level, MMSE, number of teeth) was tested using Kolmogorov-Smirnov normality Test; almost all variables considered did not follow a normal distribution. Because of that, Mann-Whitney Test was used for analysis of difference between groups (patients with and without Dementia) in age, school level, MMSE and number of teeth. Analysis of correlation has been carried out with non-parametric Spearman Test. The level of significance was assumed to be $p<0.05$ or $p<0.01$. SPSS (IBM Corp. Armonk, NY) was used for the analysis.

\section{RESULTS}

378 individuals were visited (Tab. I). The range of age was between 59 and 104 years old, with mean age of $85.96 \pm 7.97(86.47 \pm 7.68$ in the cognitively impaired group and $85.79 \pm 8.89$ in the cognitively normal group). 357 patients (94.4\%) were 75 years old or older. The sample consisted of 298 women (78.8\%) and 80 men (21.2\%). On average, level of schooling 


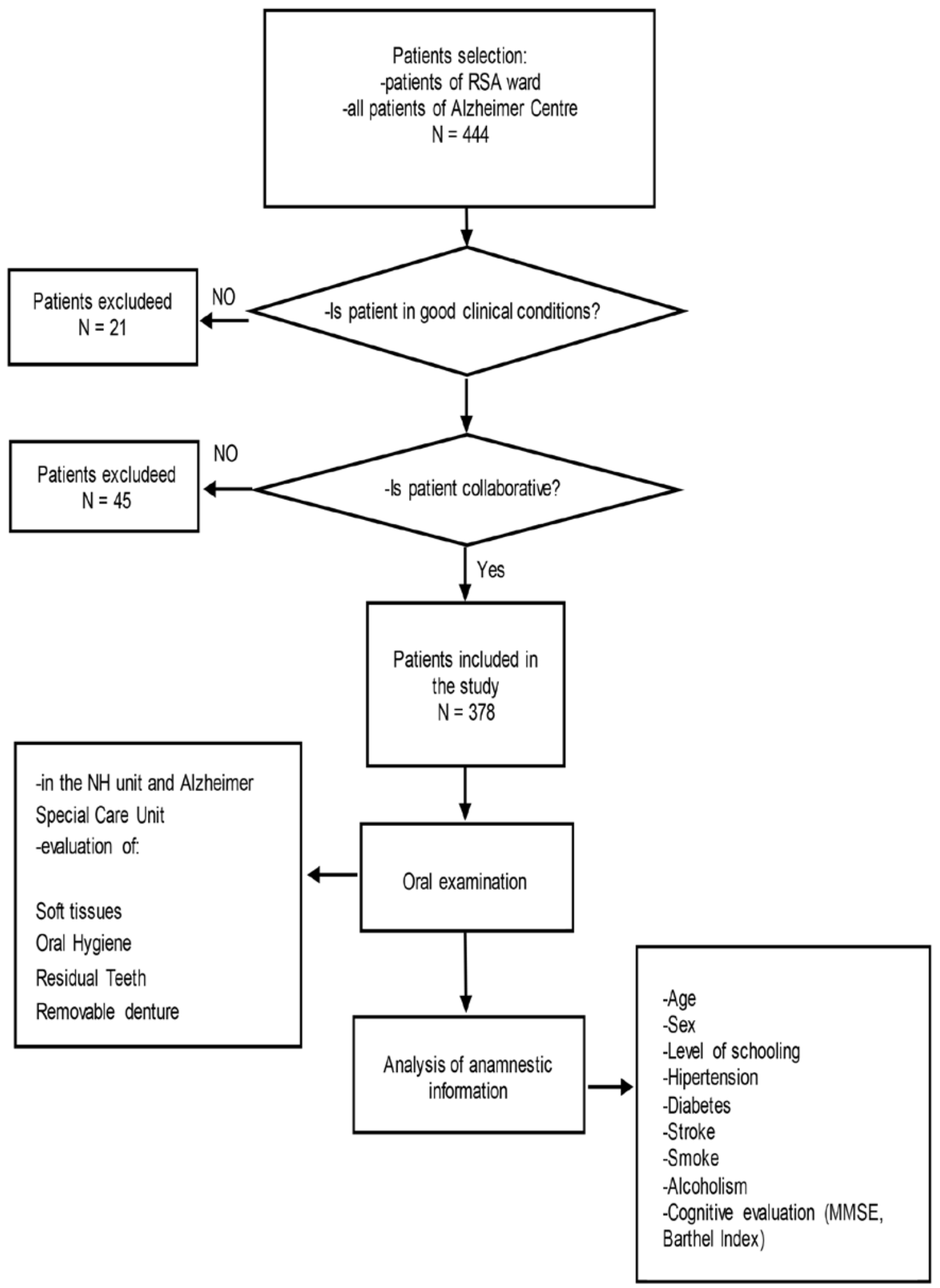

Figure 1. Flowchart. Description of the procedure for the patients'selection. 
Table I. Descriptive characteristics: anamnesis, comorbidity, cognitive status and oral conditions were used to describe the group.

\begin{tabular}{|l|c|}
\hline \multicolumn{2}{|l|}{ Characteristics } \\
\hline Anamnesis \\
\hline$\geq 75$ aa & 339 \\
\hline M & 78 \\
\hline F & 286 \\
\hline Level of schooling $\leq 10$ years & 306 \\
\hline Comorbidity & 205 \\
\hline Hypertension & 54 \\
\hline Diabetes & 33 \\
\hline Stroke & 29 \\
\hline Smoker & 8 \\
\hline Alcoholic & \\
\hline Cognitive status & 101 \\
\hline Non-formalized diagnosi & 60 \\
\hline Alzheimer disease & 144 \\
\hline Vascular dementia & 73 \\
\hline Other kind of dementia & 29 \\
\hline Residual teeth & 18 \\
\hline 0 & \\
\hline $1-9$ & 184 \\
\hline $10-19$ & 110 \\
\hline$\geq 20$ & 45 \\
\hline Mobile denture & 39 \\
\hline Totale & \\
\hline Rimovibile & \\
\hline MMSE & \\
\hline$\leq 9$ & \\
\hline $10-20$ & \\
\hline $21-26$ & \\
\hline$\geq 27$ & \\
\hline Bartehl/ADL & \\
\hline $0-24$ & \\
\hline $25-49$ & \\
\hline $50-74$ & \\
\hline $75-90$ & \\
\hline $91-100$ & \\
\hline & \\
\hline
\end{tabular}

was $7.29 \pm 3.53$ year; 306 participants (80.9\%) had a school level lower than 10 years.

277 patients (73.3\%), which previously received a formal diagnosis of dementia, were mainly in the moderate or severe stage of the disease; among them, there were 60 subjects with Alzheimer's disease, 144 with Cerebrovascular Dementia, 73 with other kind of cognitive impairment. The remaining 101 patients (26.7\%) had no formal diagnosis of dementia in their clinical file or received a diagnosis of Mild Cognitive Impairment (MCl). Among them, just according to cognitive performance, some patient could be reasonably considered in the mild stage of a dementia condition (Tab. I). On average, MMSE score was $10.67 \pm 9.03(7.20 \pm 6.85$ for subjects with dementia and $20.68 \pm 6.36$ for patients without a formal diagnosis of dementia or $\mathrm{MCl}$ ).

Across the population, 205 subjects had hypertension, 54 diabetes, 33 a history of stroke, 29 were smoker or formers smoker, 8 patients had previous history of alcoholism.

The mean number of teeth was $5.70 \pm 8.17 ; 39$ patients had more than 20 teeth left, 45 had a number of teeth between 19 and 10, 110 from 9 to 1 tooth, 184 were completely edentulous. 99 subjects 026.2\%) used regularly a denture; among them, 91 had a full removable denture, while 8 patients had a partial prosthesis.

Distribution of age, school level, MMSE and number of teeth was analysed with Kolmogorov-Smirnov Test. Because these variables did not have a normal distribution, the comparison between patients was done with Mann-Whitney Test (Tab. II). Patients with diagnosis of dementia had a significantly lower level of education than patients with no formal diagnosis of dementia or $\mathrm{MCl}(\mathrm{U}=11356.000, \mathrm{z}=-2.338, \mathrm{p}<0.05)$; similarly, MMSE score $(U=2310.000, z=-12.578, p<0.05)$ and number of teeth $(U=11210.000, z=-3,13$, $\mathrm{p}<0.05)$ were significantly lower among patients with dementia. These two groups did not differ by mean age $(U=12311.000, z=-1,786, n s)$ : on average, age was similar between patients with and without dementia. A significant negative association was found between age and number of residual teeth $\left(r_{s}=-.209, p<0.01\right)$, while MMSE score did not correlate significantly with the amount of teeth $\left(r_{s}=0.082, p=n s\right)$. There was a mild correlation between number of teeth and level of schooling $\left(r_{s}=0.149, p<0.01\right)$. A slight negative correlation was found between SOSIA score and number of teeth: the higher was the class and the level of complexity care, the lower was the number of residual teeth $\left(r_{s}=-0.111, p<0.05\right)$.

\section{DISCUSSION}

The present study allowed to evaluate general and clinical conditions and state of oral health, showing significant differences between patients with and without a formal diagnosis of cognitive impairment. Despite the average being similar in the groups, the number of teeth was lower among people with dementia, as well as level of education and, subsequently, the MMSE score. These findings are in agreement with literature: patients with dementia tend to have a lower level of education (aspect that is recognized as a risk factor) ${ }^{10}$ and lower results in cognitive test. The number of teeth appears 
Table II. Comparison: age, education, MMSE and number of teeth were used to compare patients with and without formal diagnosis of dementia. Mann-Whitney Test was used for the analysis. P-value $<0.05$. (NS = not significant).

\begin{tabular}{|c|c|c|c|c|}
\hline & & $\begin{array}{l}\text { Formal } \\
\text { diagnosis } \\
\text { of dementia } \\
(n=277)\end{array}$ & $\begin{array}{l}\text { No formal diagnosis } \\
\text { of dementia/diagnosis of } \\
\text { MCl } \\
(n=101)\end{array}$ & $\boldsymbol{P}$-value \\
\hline \multirow[t]{5}{*}{ Age } & & & & NS \\
\hline & Mean & 86,47 & 84.79 & \\
\hline & Median & 87 & 86 & \\
\hline & IQR & 7.67 & 8.89 & \\
\hline & Standard deviation & 10 & 11,25 & \\
\hline \multirow[t]{5}{*}{ Schooling } & & & & 0.019 \\
\hline & Mean & 7.022 & 8.06 & \\
\hline & Median & 5 & 5 & \\
\hline & IQR & 3.29 & 4,02 & \\
\hline & Standard deviation & 3 & 8 & \\
\hline \multirow[t]{5}{*}{ MMSE } & & & & 0.001 \\
\hline & Mean & 7.2 & 20.69 & \\
\hline & Median & 8 & 22 & \\
\hline & IQR & 6.85 & 6.36 & \\
\hline & Standard deviation & 12 & 8 & \\
\hline \multirow[t]{5}{*}{ Residual teeth } & & & & 0.002 \\
\hline & Mean & 4.64 & 8.79 & \\
\hline & Median & 0 & 5 & \\
\hline & IQR & 7.18 & 9.94 & \\
\hline & Standard deviation & 6 & 19 & \\
\hline
\end{tabular}

to be reduced in patients with dementia, either as a result of a reduced ability of maintaining hygiene or as predisposing factor ${ }^{11}$. In the studied population, very poor attention to oral hygiene was observed on the part of caregivers and a specialized monitoring was totally lacking. There was an inverse correlation between age and number of teeth: increasing the years, patients (with and without cognitive impairment) had a higher number of edentulous areas. These results can be ascribed to not only poor general physical condition of the elderly, but also to reduced ability of cleaning, that requires more support and help ${ }^{12}$.

Regardless of level of cognitive impairment, a higher degree of schooling was associated with an increased amount of remaining teeth. People with higher education, and probably higher social background, may have had access to adequate dental care since before admission, continuing to maintain good oral health when admitted in $\mathrm{NH}$.

Several studies have examined the possible mechanisms of association between tooth loss and progression of cognitive impairment ${ }^{13}$ : a reduced masticatory efficiency and the involvement of periodontal pathogens in the development of inflammatory processes of the brain seem to be, currently, the most accredited pathways. However, in the present study, no correlation between
MMSE and residual teeth number seems to emerge, perhaps due to the presence, in the studied population, of a high percentage of subjects with low MMSE score and severe cognitive impairment. Thus, the distribution of patients could have invalidate an analysis in this direction.

The constant use of denture was limited to a low percentage of patients; most subjects with partial or total edentulism did not have prosthesis or had incongruous denture, not cleaned or, in some cases, not their own. A severe cognitive impairment may have influenced, since before admission, the maintenance of proper oral hygiene and patient adaptation for eventual restoration.

It is possible that both variables (poor oral condition and inadequacy of the prosthesis) influenced and influence even during hospitalization the nutritional status of patients. Some studies have shown an association between masticatory efficiency (guaranteed by the use of implants or from natural teeth), nutrition quality and cognitive status. Optimal chewing performance positively influences not only the maintenance of an appropriate nutritional intake, but also the regulation of cerebral blood flow, helping to reduce the risk of cognitive decline ${ }^{14}$. Correlation study made it possible to identify a negative association between SOSIA score and number of teeth: 
a higher level of complexity of care is associated with a lower number of residual teeth. As already mentioned, the total or partial edentulism can be expression of poor general physical condition; on the other hand, a growing number of studies are investigating the report and the predictability of tooth loss on the onset of diseases such as diabetes, cardiovascular disease and obesity, signalling significant correlations.

The difference in number of residual teeth between patients with and without dementia might have been influenced by other factors, such as general surgical conditions and the level of education.

\section{CONCLUSIONS}

Based on a considerable sample of hospitalized patients and on a timely analysis of individual clinical history, this study highlight significant differences among subjects with a formal diagnosis of dementia and patients with $\mathrm{MCl}$ or without a formal diagnosis of dementia. Oral health conditions were very poor, with abundant deposits of plaque and tartar and badly preserved dentures, particularly among patients with advanced cognitive decline. In this group of patients, attention to hygienic aspect is poor, with possible repercussions on the quality of life (self-perception, socialization, relationship with neighbours) and nutritional status. This underestimation of the oral cavity hygiene should be considered and examined in depth, in order to improve the competence of caregivers. Despite the limits of this study (presence of patients with advanced cognitive decline, absence of a control group), we can still get a confirmation of the relationship between oral conditions and dementia, in line with the literature ${ }^{15}$. Correlations among the number of teeth and level of cognitive impairment with other variables should be taken into account and analysed, including comorbidity and socio-economic conditions, which could play a potential confounding role.

\section{References}

1 World Alzheimer Report 2018. The state of the art of dementia research: new frontiers. ADI 2018 (https://www.alz. co.uk/research/WorldAlzheimerReport2018.pdf).

2 Zimmerman S, Anderson WL, Brode S, et al. Systematic review: effective characteristics of nursing homes and other residential long-term care settings for people with dementia. J
Am Geriatr Soc 2013;61:1399-409. https://doi.org/10.1111/ jgs. 12372

3 On B, Han DH, Han KT, et al. Association between residual teeth number in later life and incidence of dementia: a systematic review and meta-analysis. BMC geriatrics 2018;18:4857. https://doi.org/10.1186/s12877-018-0729-z

4 Harding A, Robinson SJ, Crean S, et al. Can better management of periodontal disease delay the onset and progression of Alzheimer's disease? JAD 2017;58:337348. https://doi. org/10.3233/JAD-170046

5 Felton D. Complete edentulism and comorbid diseases: an update. J Prosthodontics 2016;25:5-20. https://doi. org/10.1111/jopr.12350

6 Takeuchi K, Ohara T, Furuta M, et al. Tooth loss and risk of dementia in the community: the Hisayama study. J Am Geriatri Soc 2017;65:95-100. https://doi.org/10.1111/jgs.14791

7 Saito S, Ohi T, Murakami T, et al. Association between tooth loss and cognitive impairment in community-dwelling older Japanese adults: a 4-year prospective cohort study from the Ohasama study. BMC Oral Health 2018;18:142-50. https:// doi.org/10.1186/s12903-018-0602-7

8 Dotti C, Casale G, Zacchi V, et al. La classificazione SOSIA degli anziani ospiti delle residenze sanitario-assistenziali lombarde. Ann lg 2006;18:439-51.

9 American Psychiatric Association, DSM-IV-TR MG. Diagnostic and statistical manual of mental disorders. Milano: Elsevier 1996, pp. 27, 82, 83, 86-88, 92.

10 Schwindling FS, Krisam J, Hassel AJ, et al. Long-term success of oral health intervention among care-dependent institutionalized seniors: findings from a controlled clinical trial. Community Dent Oral Epidemiol 2018;46:109-17. https:// doi.org/10.1111/cdoe.12335

11 Ishimiya M, Nakamura H, Kobayashi $\mathrm{Y}$, et al. Tooth lossrelated dietary patterns and cognitive impairment in an elderly Japanese population: the Nakajima study. PLoS One 2018;13:e0194504. https://doi.org/10.1371/journal. pone.0194504

12 Kossioni AE. The association of poor oral health parameters with malnutrition in older adults: a review considering the potential implications for cognitive impairment. Nutrients 2018;10:1709. https://doi.org/10.3390/nu10111709

13 Tonsekar PP, Jiang SS, Yue G. Periodontal disease, tooth loss and dementia: Is there a link? A systematic review. Gerodontology 2017;34:151-63. https://doi.org/10.1111/ger.12261

14 Lin C. Revisiting the link between cognitive decline and masticatory dysfunction. BMC geriatrics 2018;18:5-20. https://doi. org/10.1186/s12877-017-0693-z

15 Fang WL, Jiang MJ, Gu BB, et al. Tooth loss as a risk factor for dementia: systematic review and meta-analysis of 21 observational studies. BMC Psychiatry 2018;18:345-56. https://doi.org/10.1186/s12888-018-1927-0 